\title{
Functional connectivity changes between parietal and prefrontal cortices in primary insomnia patients: evidence from resting-state fMRI
}

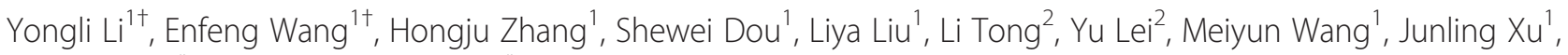
Dapeng Shi ${ }^{*}$ and Qingyong Zhang ${ }^{3^{*}}$

\begin{abstract}
Background: Primary insomnia can severely impair daytime function by disrupting attention and working memory and imposes a danger to self and others by increasing the risk of accidents. We speculated that the neurobiological changes impeding working memory in primary insomnia patients would be revealed by resting-state functional MRI (R-fMRI), which estimates the strength of cortical pathways by measuring local and regional correlations in blood oxygen level dependent (BOLD) signs independent of specific task demands.
\end{abstract}

Methods: We compared the R-fMRI activity patterns of 15 healthy controls to 15 primary insomnia patients (all 30 participants were right-handed) using a 3.0 T MRI scanner. The SPM8 and REST1.7 software packages were used for preprocessing and analysis. Activity was expressed relative to the superior parietal lobe (SPL, the seed region) to reveal differences in functional connectivity to other cortical regions implicated in spatial working memory.

Result: In healthy controls, bilateral SPL activity was associated with activity in the posterior cingulate gyrus, precuneus, ventromedial prefrontal cortex, and superior frontal gyrus, indicating functional connectivity between these regions. Strong functional connectivity between the SPL and bilateral pre-motor cortex, bilateral supplementary motor cortex, and left dorsolateral prefrontal cortex was observed in both the control group and the primary insomnia group. However, the strength of several other functional connectivity pathways to the SPL exhibited significant group differences. Compared to healthy controls, connectivity in the primary insomnia group was stronger between the bilateral SPL and the right ventral anterior cingulate cortex, left ventral posterior cingulate cortex, right splenium of the corpus callosum, right pars triangularis (right inferior frontal gyrus/Broca's area), and right insular lobe, while connectivity was weaker between the SPL and right superior frontal gyrus (dorsolateral prefrontal cortex).

Conclusion: Primary insomnia appears to alter the functional connectivity between the parietal and frontal lobes, cortical structures critical for spatial and verbal working memory.

Keyword: Functional MRI, Primary insomnia, Working memory, Superior parietal lobule

\section{Background}

Chronic insomnia is the most common sleep disorder, afflicting 10 to $20 \%$ of the adult population worldwide $[1,2]$. It is defined by the Diagnostic and Statistical Manual of Mental Disorders, version 4 (DSM-IV) as both an independent psychiatric syndrome (primary insomnia) and

\footnotetext{
*Correspondence: shidapeng0904@126.com; qyzhang@zzu.edu.cn ${ }^{\dagger}$ Equal contributors

'Department of Radiology, Henan Provincial People's Hospital, People's Hospital of Zhengzhou University, Zhengzhou, Henan 450003, China ${ }^{3}$ Second affiliated hospital of Zhengzhou University, Zhengzhou, Henan, China Full list of author information is available at the end of the article
}

a common comorbidity (secondary insomnia) associated with a variety of physical and psychiatric disorders [3]. According to a global insomnia survey by the Chinese Medical Association (CMA), 42.5\% of people in China suffer from occasional insomnia, and rates are increasing due to industrialization, urbanization, and work pressures. Difficulty falling asleep and staying asleep reduces quality of life [4]. The most salient effect of insomnia is increased daytime sleepiness, resulting in impaired concentration, cognition, and memory [5]. Moreover, insomnia patients reported more missed work days and 
greater utilization of the healthcare system, even when factoring in the higher prevalence of depression and chronic medical conditions [6]. While both pharmacological and behavioral therapies have demonstrated efficacy for the treatment of insomnia, drugs for insomnia carry potential risks of overdose and dependency, while cognitive behavior therapies are expensive, time consuming, and not widely accessible by many sufferers [7]. Development of safer or more practical alternatives requires further insight into the neurobiological changes associated with chronic sleep deprivation.

Sleep is critical for brain development and function [8-10]. Working memory (WM), a core component of executive function, is markedly disrupted by sleep deprivation [11-13]. Neural circuits associated with emotional regulation and expression are markedly altered in insomnia patients as revealed by functional magnetic resonance imaging (fMRI) [14]. Resting- state functional magnetic resonance imaging ( $\mathrm{R}-\mathrm{fMRI}$ ) revealed reduced amygdala connectivity to the insula, striatum, and thalamus, as well as enhanced functional connectivity with the premotor and sensorimotor cortex, suggesting potential neurological mechanisms for dysregulated emotional control and affective disorders associated with insomnia $[15,16]$. Sleep deprivation also triggers compensatory mechanisms to overcome fatigue that are manifested by changes in neural connectivity patterns on fMRI [17].

The prevalence of secondary insomnia may be severalfold greater than primary insomnia [18] and symptoms more severe [19]. However, associated neuropsychiatric comorbidities are manifested by a variety of changes in brain physiology and functional organization [20,21], so only studies of primary insomnia can reveal changes in functional connectivity associated specifically with sleep deprivation. Resting-state fMRI (R-fMRI) measures changes in neural connectivity patterns unbiased by the demands of specific cognitive tasks [22,23]. Thus, R-fMRI is optimal for foundational studies on the anatomic and functional alterations associated with insomnia. Moreover, functional connectivity patterns defined by R-fMRI are well correlated with task-dependent activity patterns [24]. As WM is essential for daily function and markedly degraded by sleep deprivation, we examined changes in functional cortical circuits implicated in WM using RfMRI. The parietal lobe is an important structure for spatial working memory (SWM) as indicated by BOLD signals during SWM tasks in healthy controls [25], and so was chosen as the seed regions for this study.

\section{Method}

The study procedures were approved by the Zhengzhou University ethics committee and all subjects provided written informed consent. We compared the R-fMRI of 15 healthy right-handed undergraduate students or hospital faculty staff from Zhengzhou University Medical School and Hospital (mean age \pm SD: $39.8 \pm 11.2$ years; age range 21 to 65 years, 8 females and 7 males) to ageand gender ratio-matched right-handed subjects meeting DSM-IV inclusion criteria for primary insomnia $(41.3 \pm 8.9$ years; age range 23 to 54 years, 8 females and 7 males) [3]. Both primary insomnia patients and healthy controls were evaluated using the Pittsburg Sleep Quality Index (PSQI) [26]. All healthy controls reported their sleep as restorative and satisfactory, and all had regular sleep habits with PSQI total scores $<5$. Both cohorts were also screened to ensure that they had no history of chronic medical or psychiatric illnesses and had never been medicated for insomnia or suffered other sleep disorders. No participant habitually consumed more than $250 \mathrm{mg} /$ day caffeine. All participants were instructed to rest for 30 minutes prior to the experiment and not to consume caffeine, alcohol, or any other psychoactive substances for 24 hours before the R-fMRI study.

\section{Data Acquisition}

Functional imaging was conducted on a Siemens 3.0 T TrioTim whole-body scanner (Siemens AG, Erlangen, Germany) using a 12-channel array coil in the Henan Provincial People's Hospital.

High-resolution T1-weighted anatomic images were obtained using a 3D-MPRAGE sequence $(T R=1,950 \mathrm{~ms}$, $\mathrm{TE}=2.30 \mathrm{~ms}, \mathrm{Ti}=900 \mathrm{~ms}$, scan time $=4.24$ minutes, matrix $=248 \times 256$, slice thickness $=1 \mathrm{~mm}$, no distance, FOV $=244 \times 252)$ and fMRI images using a BOLD-fMRI sequence $(\mathrm{TR}=3,000 \mathrm{~ms}, \mathrm{TE}=2.50 \mathrm{~ms}$, matrix $=320 \times$ 320 , slice thickness $=5 \mathrm{~mm}$, slice interval $=0.5 \mathrm{~mm}$, total layer $=35, \mathrm{FOV}=210 \times 210$, scan time $=7$ minutes). The entire brain was scanned in 140 volumes. Subjects were instructed to keep their eyes closed, relax, and remain calm during the resting-state scan.

\section{Functional MRI data analysis processing Data pre-processing}

Pre-processing was performed using SPM8 (SPM8, http:// www.fil.ion.ucl.ac.uk/spm) and REST (Resting-state data analysis toolkit, http:/www.restfmri.net/forum/REST) programs. All functional runs were expressed relative to the first values in each run. We set a movement threshold of $1.5 \mathrm{~mm}$ and $1.5^{\circ}$ for the 3 linear and 3 axial coordinates to eliminate subjects with excessive head movement. However, none of the subjects had head movements that exceeded threshold. All functional runs were normalized to Montreal Neurological Institute (MNI) space with voxel re-sampling to $3 \times 3 \times 3 \mathrm{~mm}^{3}$. After spatial normalization, we used REST to extract the linear changes over time within the 0.01 to $0.08 \mathrm{~Hz}$ bandwidth. The resulting time 
series were then spatially smoothed with a 4-mm fullwidth at half maximum (FWHM) Gaussian kernel.

\section{Functional connectivity analysis}

The parietal lobe plays a dominant role in spatial information processing [27] and so was chosen as the seed region to determine changes in functional connectivity patterns by R-fMRI. The exact region of interest (ROI) within the bilateral superior parietal lobe was chosen using WFU_PickAtlas (http://www.ansir.wfubmc.edu). Voxels within the seed region were averaged to generate reference time series. For each subject, a correlation map was then produced by computing the Pearson's correlation coefficients between the reference time series and the time series of all other brain voxels. The Fisher transformation was used to compute the z-values from $\mathrm{r}$-values in order to improve the normality. Individual $z$-values for each voxel outside the SPL were compared to that of the SPL seed region ROI by one-sample $t$-test to reveal brain regions showing significant connectivity to the bilateral SPL. Clusters larger than $405 \mathrm{~mm}^{3}$ with $P<0.005$ were accepted and used to construct functional connectivity images.

\section{Statistical analysis}

The z-values were also entered into a random effect two sample $t$-test to identify regions showing significant differences in connectivity relative to the bilateral superior parietal lobes between primary insomnia patients and healthy controls. We set $P<0.05$ as a significant difference threshold.

\section{Results}

We examined differences in connectivity to the bilateral superior parietal lobe (SPL) between a healthy control group and a primary insomnia patient group using resting-state fMRI (R-fMRI). In the healthy control group, there were strong correlations between SPL and posterior cingulate and the nearby precuneus, ventromedial prefrontal gyrus, and superior frontal gyrus (Figure 1). Resting-state activities of bilateral pre-motor cortex, supplementary motor cortex, and left dorsolateral prefrontal cortex were strongly correlated with resting- state SPL activity in both the primary insomnia patient group and the control group (Figures 1, 2). Several regions, however, exhibited significant group differences in functional connectivity to the SPL as revealed by two sample $t$-tests. Compared to the healthy control group, the primary insomnia patient group showed significantly increased functional connectivity between the SPL and bilateral anterior and posterior cingulate, splenium of the corpus callosum, right middle frontal gyrus, and right claustrum (Figure 3 and Table 1). In contrast, the functional connectivity between the SPL and superior frontal gyrus was lower in primary insomnia patients (Figure 4 and Table 1).

\section{Discussion and conclusions}

Current MRI research on primary insomnia has focused on emotional circuit dysfunction [14] and changes in

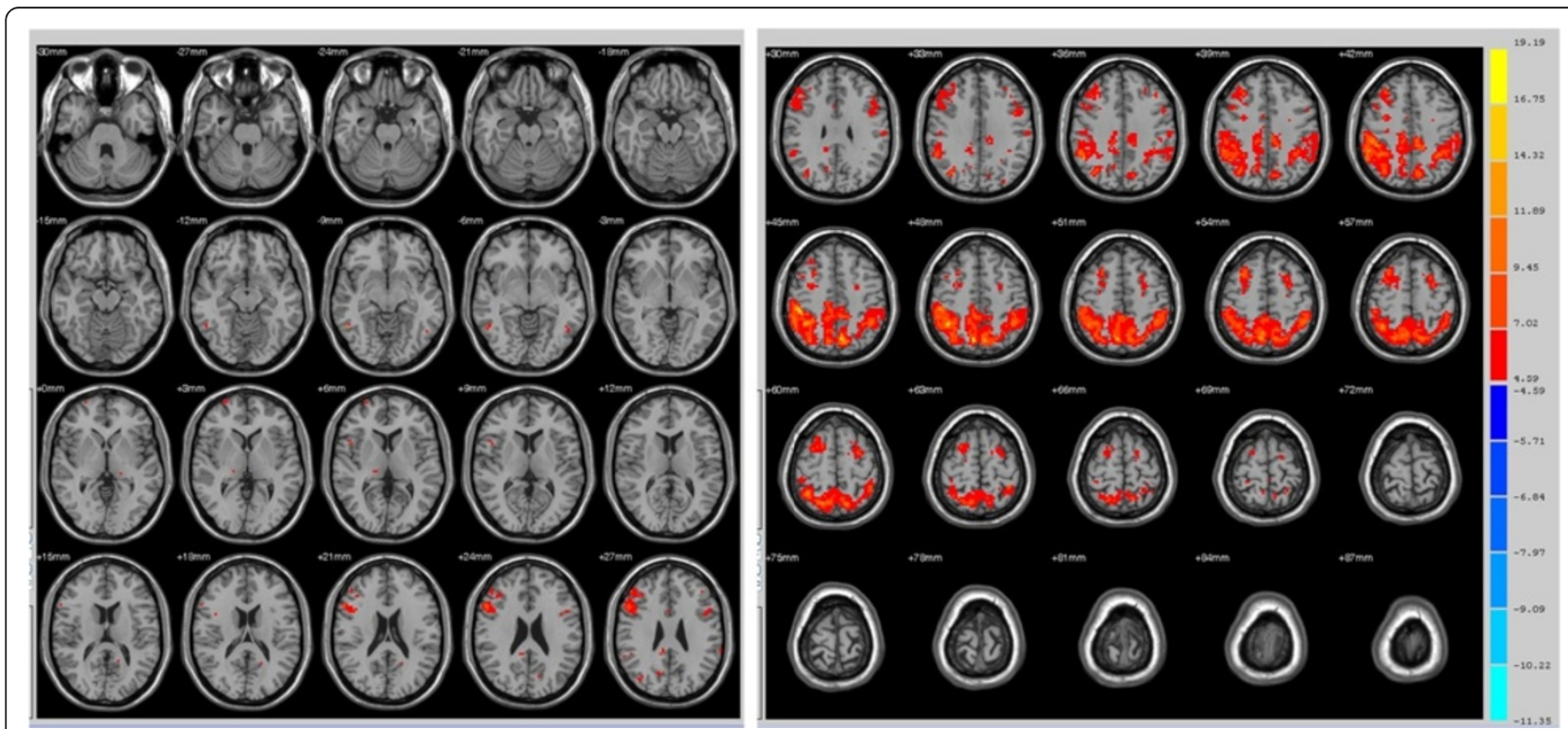

Figure 1 Maps of resting-state connectivity to the bilateral superior parietal lobe (SPL) in healthy controls. Colored areas are those regions with a time series correlation relative to the SPL of $P<0.005$ and minimum cluster size of $405 \mathrm{~mm}^{3}$. The right fusiform gyrus, right anterior prefrontal cortex, right somatosensory association cortex, bilateral pre-motor cortex, bilateral supplementary motor cortex, and left dorsolateral prefrontal cortex exhibited strong functional connectivity with the bilateral SPL. 

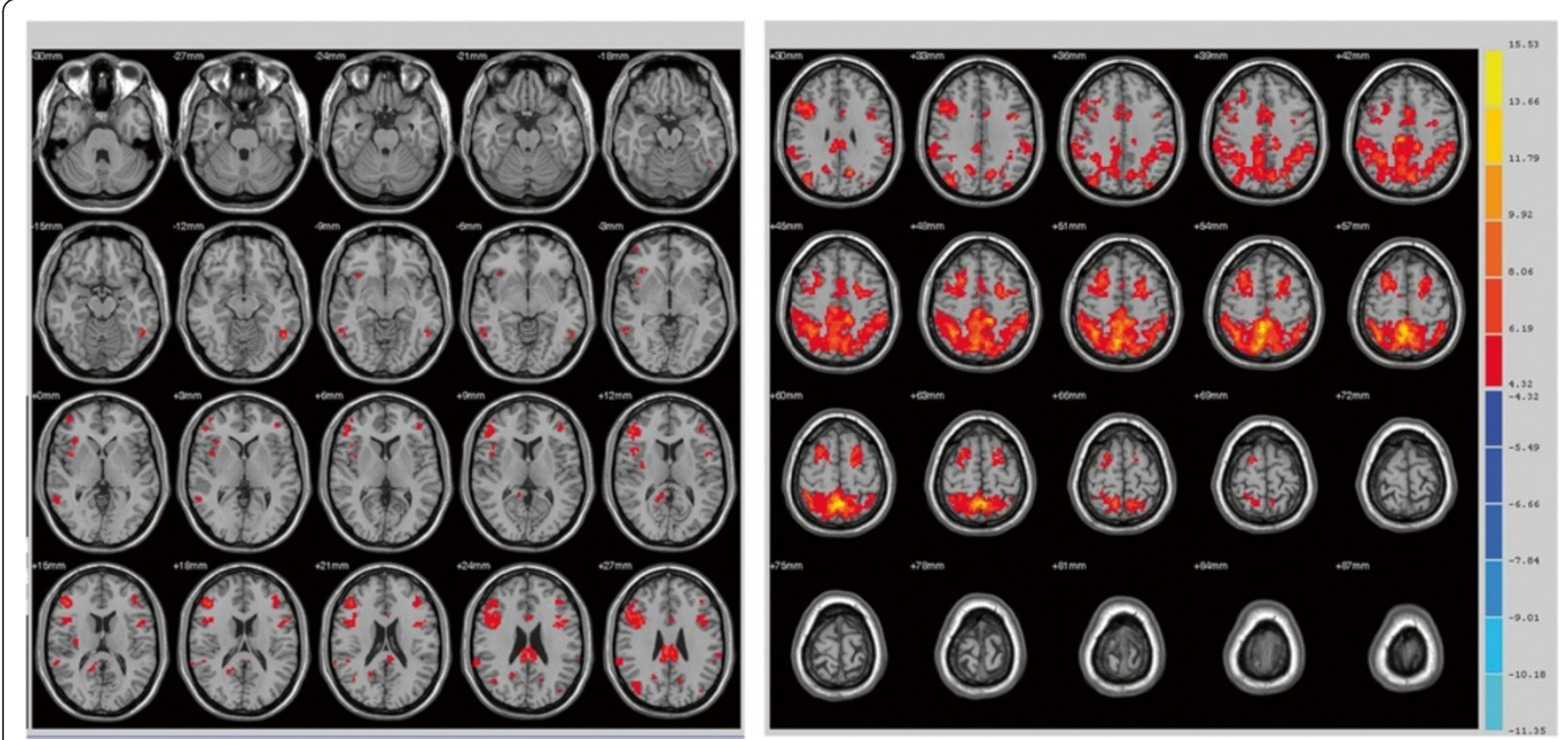

Figure 2 Maps of resting-state connectivity to the bilateral superior parietal lobe (SPL) in primary insomnia patients. Colored areas are those regions with a time series correlation relative to the SPL of $P<0.005$ and a minimum cluster size of $405 \mathrm{~mm}^{3}$. The right superior temporal gyrus, right pars triangularis (inferior frontal gyrus/Broca's area), right dorsolateral prefrontal cortex, right insular cortex, bilateral pre-motor cortex, bilateral supplementary motor cortex, left fusiform gyrus, and left dorsolateral prefrontal cortex exhibited strong functional connectivity with the bilateral SPL.

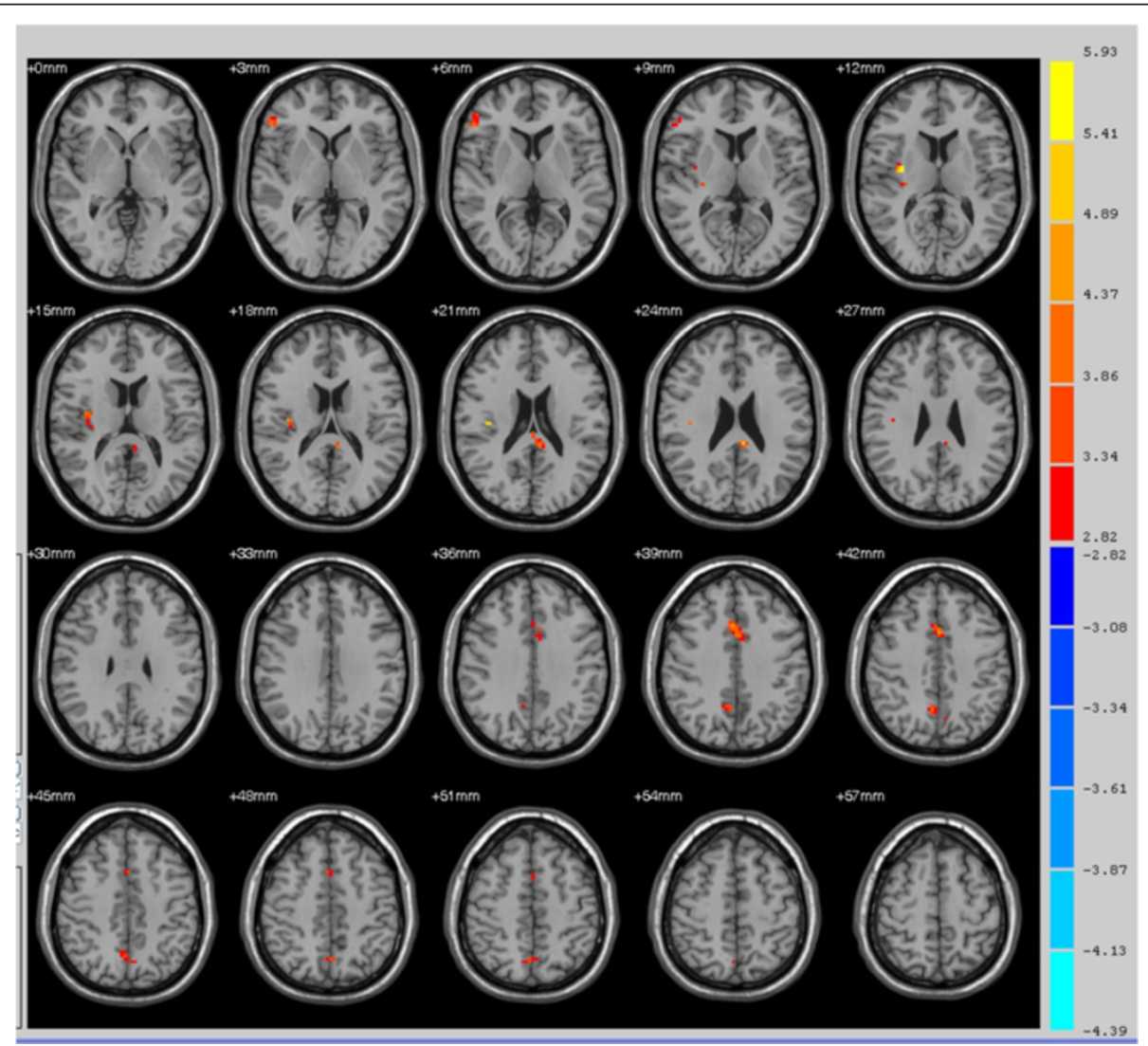

Figure 3 Areas with higher functional connectivity to the bilateral superior parietal lobe in primary insomnia patients compared to healthy controls. Areas of greater functional connectivity in patients include the right pars triangularis (inferior frontal gyrus/Broca's area, thin blue arrow), right insular cortex (thick blue arrow), right anterior cingulate cortex (thin red arrow), and left ventral posterior cingulate cortex (thick red arrow) (all $P<0.01$ ). 
Table 1 Functional connectivity differences between the healthy and primary insomnia groups

\begin{tabular}{|c|c|c|c|c|c|c|c|}
\hline \multirow[t]{2}{*}{ Cortical regions } & \multirow[t]{2}{*}{ BA } & \multirow[t]{2}{*}{ Clusters } & \multicolumn{3}{|c|}{$\begin{array}{l}\text { MNI } \\
\text { coordinate }\end{array}$} & \multirow[t]{2}{*}{$t$-values } & \multirow[t]{2}{*}{$P$-value } \\
\hline & & & $x$ & $\mathrm{Y}$ & Z & & \\
\hline $\begin{array}{l}\text { Right pars triangularis, } \\
\text { part of Broca's area }\end{array}$ & 45 & 20 & 54 & 32 & 4 & 3.6824 & 0.01 \\
\hline Right insular cortex & 13 & 26 & 33 & -7 & 13 & 4.7183 & 0.01 \\
\hline $\begin{array}{l}\text { Left ventral posterior } \\
\text { cingulate cortex }\end{array}$ & 23 & 18 & -6 & -37 & 25 & 4.0944 & 0.01 \\
\hline $\begin{array}{l}\text { Right ventral anterior } \\
\text { cingulate cortex }\end{array}$ & 24 & 39 & 3 & 17 & 40 & 4.0641 & 0.01 \\
\hline $\begin{array}{l}\text { Right dorsal posterior } \\
\text { cingulate cortex }\end{array}$ & 31 & 31 & 6 & -58 & 43 & 3.5088 & 0.01 \\
\hline $\begin{array}{l}\text { Right dorsolateral } \\
\text { prefrontal cortex }\end{array}$ & 46 & 21 & 51 & 38 & 28 & -4.3949 & 0.05 \\
\hline
\end{tabular}

BA, Brodmann area; MNI: Montreal Neurological Institute.

pathways mediating hyperarousal [28]. Huang et al. [14] found reduced connectivity of the amygdala to the insula, striatum, and thalamus by R-fMRI, but increase amygdalar connectivity to the pre-motor and sensorimotor cortices in primary insomnia patients. These changes in emotional circuits may contribute to the ultimate development of comorbidities such as depression and anxiety disorder, while the increased functional connectivity of the amygdala to the pre-motor and sensorimotor cortices may be compensatory mechanisms to maintain psychomotor performance under increased daytime fatigue [14]. It has long been speculated that insomnia results from psychological hyperarousal by stressors and, in fact, insomnia patients exhibited enhanced functional connectivity among various sensory cortices and supplementary motor cortex (SMC) as revealed by R-fMRI, and sleep initiation difficulties were associated with the strength of sensory-SMC connectivity [28]. Despite the fact that insomnia is known to markedly impair WM and other aspects of executive function, there have been no studies on possible connectivity changes associated with SWM dysfunction in primary insomnia patients. We demonstrate clearly reduced functional connectivity between the SPL and superior frontal gyrus, which may underlie spatial and verbal WM deficits resulting from insomnia.

SWM tasks activate the hippocampus, the cingulate gyrus, prefrontal dorsolateral cortex, occipitotemporal junction cortex, and parietal cortices [29-34], and SWM depends critically on the interaction between frontal and parietal regions [35]. Indeed, when the superior parietal

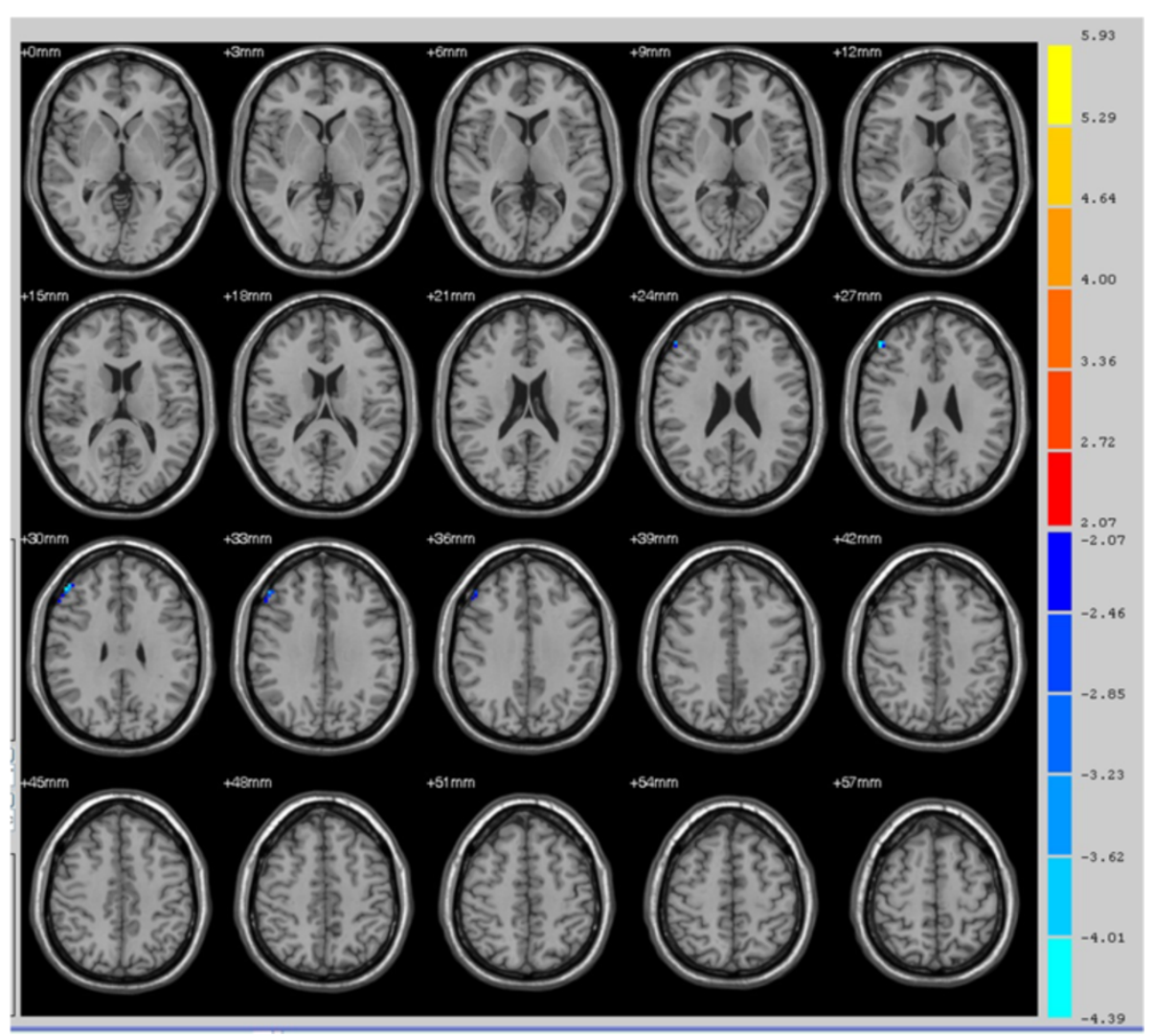

Figure 4 Areas with lower resting-state connectivity to the bilateral superior parietal lobe in primary insomnia patients compared to healthy controls, including the right superior frontal gyrus (right dorsolateral prefrontal cortex, red arrow) $(P<0.05)$. 
lobes are damaged, sense of position and directional motion are lost, while unilateral right parietal lobe damage is associated with contralateral neglect [27]. The mammalian parietal medial surface and dorsolateral superior parietal region project to and receive fibers from the prefrontal cortex. The parietal region Brodmann area (BA) 7, which is involved in integration of visual and proprioceptive information, and BA 46 in the prefrontal cortex have particularly strong bilateral connections. The dorsal prefrontal lobe receives association fibers from the parietal lobe encoding dynamic visual information on position and space between objects. In the prefrontal lobe, these inputs are integrated to form spatial working memories that aid in various cognitive processes [36]. In addition to SWM, these two regions are also critical for verbal WM [37].

In our study, we measured strong connections between the SPL and the posterior cingulate gyrus (PCC), precuneus, ventromedial prefrontal cortex, and superior frontal gyrus in healthy controls. These areas were also strongly connected in primary insomnia patients with the exception of the superior frontal gyrus. In contrast, the functional connectivity between the anterior cingulate cortex (ACC) and SPL was stronger in primary insomnia patients. It is agreed that the prefrontal lobe subserves WM and that the superior prefrontal cortex stores information on spatial position [32-34]. Primary insomnia patients had lower functional connectivity between the superior frontal gyrus and SPL, consistent with the SWM deficits observed in these patients. Further research using complementary R-fMRI and fMRI during SWM tasks could reveal additional insights into the neurobiology of WM deficits due to insomnia.

In conclusion, our study strongly suggests that primary insomnia induces memory retrieval deficits in human subjects by disrupting the functional connectivity between the superior frontal gyrus and superior parietal lobe.

\section{Abbreviations}

BA: Brodmann area; BOLD: blood oxygen level dependent; CMA: Chinese Medical Association; fMRI: functional magnetic resonance imaging; FWHM: fullwidth at half maximum; MNI: Montreal Neurological Institute; PSQI: Pittsburg Sleep Quality Index; R-fMRI: resting- state functional MRI; SPW: superior parietal lobe; SWM: spatial working memory; WM: Working memory.

\section{Competing interests}

The authors declare that they have no competing interests.

\section{Authors' contributions}

DS, QZ and YL made substantial contributions to study conception and design as well as data acquisition and analysis, drafting the manuscript or revising it critically for important intellectual content, and gave final approval for publication. EW, SD, and LL were involved in data acquisition, analysis, and interpretation. $\mathrm{HZ}$ helped screen primary insomnia patients and healthy controls. LT, YL, M W, and JX were involved in analysis and interpretation of data. All authors read and approved the final manuscript.

\section{Acknowledgements}

This research was partially supported by 2013 Henan Provincial Key Technological Projects of Department of Science \& Technology, study on the neural network of cerebral function imaging in impaired spatial working memory of primary insomnia, No. 1321023100633.and 2012 Henan Provincial Key Technological Projects of Department of Public Health, study on the pre- and post-treatment functional magnetic resonance imaging in primary insomnia patients, No. 201202023 and National Key Specialty Projects.

\section{Author details}

'Department of Radiology, Henan Provincial People's Hospital, People's Hospital of Zhengzhou University, Zhengzhou, Henan 450003, China. ${ }^{2}$ China National Digital Switching System Engineering and Technological Research Center, Zhengzhou, Henan, China. ${ }^{3}$ Second affiliated hospital of Zhengzhou University, Zhengzhou, Henan, China.

Received: 6 November 2013 Accepted: 15 May 2014 Published: 10 June 2014

\section{References}

1. Buysse DJ: Insomnia. JAMA 2013, 309:706-716.

2. Kraus SS, Rabin LA: Sleep America: managing the crisis of adult chronic insomnia and associated conditions. J Affect Disord 2012, 138:192-212.

3. Diagnostic and Statistical Manual of Mental Disorders. 4th edition. Washington DC: American Psychiatric Association.

4. Zammit GK, Weiner J, Damato N, Sillup GP, McMillan CA: Quality of life in people with insomnia. Sleep 1999, 22(Suppl 2):S379-385.

5. Carey TJ, Moul DE, Pilkonis P, Germain A, Buysse DJ: Focusing on the experience of insomnia. Behav Sleep Med 2005, 3:73-86.

6. Simon GE, VonKorff M: Prevalence, burden, and treatment of insomnia in primary care. Am J Psychiatry 1997, 154:1417-1423.

7. Morin CM, Benca R: Chronic insomnia. Lancet 2012, 379:1129-1141

8. Colrain IM: Sleep and the brain. Neuropsychol Rev 2011, 21:1-4.

9. Riemann D, Kloepfer C, Berger M: Functional and structural brain alterations in insomnia: implications for pathophysiology. Eur J Neurosci 2009, 29:1754-1760.

10. Bootzin RR, Epstein DR: Understanding and treating insomnia. Annu Rev Clin Psychol 2011, 7:435-458.

11. Diamond A: Executive functions. Annu Rev Psychol 2013, 64:135-168.

12. Nissen C, Kloepfer C, Feige B, Piosczyk H, Spiegelhalder K, Voderholzer U, Riemann D: Sleep-related memory consolidation in primary insomnia. J Sleep Res 2011, 20:129-136.

13. Walker MP: Cognitive consequences of sleep and sleep loss. Sleep Med 2008, 9(Suppl 1):S29-34.

14. Huang Z, Liang P, Jia X, Zhan S, Li N, Ding Y, Lu J, Wang Y, Li K: Abnormal amygdala connectivity in patients with primary insomnia: evidence from resting state fMRI. Eur J Radiol 2012, 81:1288-1295.

15. Baglioni C, Riemann D: Is chronic insomnia a precursor to major depression? Epidemiological and biological findings. Curr Psychiatry Rep 2012, 14:511-518.

16. Schmidt RE, Harvey AG, Van der Linden M: Cognitive and affective control in insomnia. Front Psychol 2011, 2:349.

17. Varkevisser M, Van Dongen HP, Van Amsterdam JG, Kerkhof GA: Chronic insomnia and daytime functioning: an ambulatory assessment. Behav Sleep Med 2007, 5:279-296.

18. Ohayon MM: Prevalence of DSM-IV diagnostic criteria of insomnia: distinguishing insomnia related to mental disorders from sleep disorders. J Psychiatr Res 1997, 31:333-346.

19. Espie CA, Kyle SD, Hames P, Cyhlarova E, Benzeval M: The daytime impact of DSM-5 insomnia disorder: comparative analysis of insomnia subtypes from the Great British Sleep Survey. J Clin Psychiatry 2012, 73:e1478-484.

20. Biswal BB: Resting state fMRI: a personal history. Neuroimage 2012, 62:938-944.

21. Rosazza C, Minati L: Resting-state brain networks: literature review and clinical applications. Neurol Sci 2011, 32:773-785.

22. Lee MH, Smyser CD, Shimony JS: Resting-state fMRI: a review of methods and clinical applications. AJNR Am J Neuroradiol 2012, 34:1866-1872.

23. Deco G, Jirsa VK, McIntosh AR: Emerging concepts for the dynamical organization of resting-state activity in the brain. Nat Rev Neurosci 2011, 12:43-56. 
24. Zou Q, Ross TJ, Gu H, Geng X, Zuo XN, Hong LE, Gao JH, Stein EA, Zang YF, Yang $Y$ : Intrinsic resting-state activity predicts working memory brain activation and behavioral performance. Hum Brain Mapp 2013, 34:3204-3215.

25. Jahn G, Wendt J, Lotze M, Papenmeier F, Huff M: Brain activation during spatial updating and attentive tracking of moving targets. Brain Cogn 2012, 78:105-113.

26. Buysse DJ, Reynolds CF, Monk TH, Berman SR, Kupfer DJ: The Pittsburgh Sleep Quality Index (PSQI): A new instrument for psychiatric research and practice. Psychiatry Res 1989, 28:193-213.

27. Malhotra P, Jager HR, Parton A, Greenwood R, Playford ED, Brown MM, Driver J, Husain M: Spatial working memory capacity in unilateral neglect. Brain 2005, 128:424-435.

28. Killgore WD, Schwab ZJ, Kipman M, Deldonno SR, Weber M: Insomniarelated complaints correlate with functional connectivity between sensory-motor regions. Neuroreport 2013, 24:233-240.

29. Corbetta M, Kincade JM, Shulman GL: Neural systems for visual orienting and their relationships to spatial working memory. J Cogn Neurosci 2002, 14:508-523.

30. Shipman SL, Astur RS: Factors affecting the hippocampal BOLD response during spatial memory. Behav Brain Res 2008, 187:433-441.

31. Spiers HJ, Maguire EA: The neuroscience of remote spatial memory: a tale of two cities. Neuroscience 2007, 149:7-27.

32. Menon V, White CD, Eliez $\mathrm{S}$, Glover GH, Reiss AL: Analysis of a distributed neural system involved in spatial information, novelty, and memory processing. Hum Brain Mapp 2000, 11:117-129.

33. Ricciardi E, Bonino D, Gentili C, Sani L, Pietrini P, Vecchi T: Neural correlates of spatial working memory in humans: a functional magnetic resonance imaging study comparing visual and tactile processes. Neuroscience 2006, 139:339-349.

34. Curtis CE: Prefrontal and parietal contributions to spatial working memory. Neuroscience 2006, 139:173-180.

35. Walter H, Bretschneider V, Gron G, Zurowski B, Wunderlich AP, Tomczak R, Spitzer M: Evidence for quantitative domain dominance for verbal and spatial working memory in frontal and parietal cortex. Cortex 2003, 39:897-911.

36. Rao SC, Rainer G, Miller EK: Integration of what and where in the primate prefrontal cortex. Science 1997, 276:821-824.

37. Sala JB, Rama P, Courtney SM: Functional topography of a distributed neural system for spatial and nonspatial information maintenance in working memory. Neuropsychologia 2003, 41:341-356.

doi:10.1186/2047-783X-19-32

Cite this article as: Li et al:: Functional connectivity changes between parietal and prefrontal cortices in primary insomnia patients: evidence from resting-state fMRI. European Journal of Medical Research 2014 19:32.

\section{Submit your next manuscript to BioMed Central and take full advantage of:}

- Convenient online submission

- Thorough peer review

- No space constraints or color figure charges

- Immediate publication on acceptance

- Inclusion in PubMed, CAS, Scopus and Google Scholar

- Research which is freely available for redistribution

Submit your manuscript at www.biomedcentral.com/submit 